\title{
Pressão arterial, frequência cardíaca e duplo-produto em séries sucessivas do exercício de força com diferentes intervalos de recuperação
}

\author{
Marcos D. Polito ${ }^{1}$ \\ Roberto Simão ${ }^{1}$ \\ Antônio C.L. Nóbrega ${ }^{2}$ \\ Paulo T.V. Farinatti ${ }^{1}$
}

https://doi.org/10.5628/rpcd.04.03.07

\section{RESUMO}

As respostas cardiovasculares durante o exercício de força constituem aspecto importante da segurança na actividade. O objectivo do estudo foi verificar o comportamento das pressões arteriais sistólica (PAS) e diastólica (PAD), frequência cardíaca (FC) e duplo-produto (DP) durante quatro séries de oito repetições máximas de extensão unilateral do joelho, realizadas com intervalos de recuperação de um (G1) e dois minutos (G2). Participaram 10 homens saudáveis, voluntários e experientes no treinamento de força. PAS, PAD e FC foram medidas pela técnica fotopletismográfica (Finapres') no repouso, ao final de cada série e nos dois minutos subsequentes ao término do exercício. A ANOVA de duas entradas identificou valores significativamente mais elevados em G1 que em G2, respectivamente, para PAS (mmHg) na $2^{\mathrm{a}}(166,7 \pm 19,9$ vs. $147,7 \pm 13,5)$, $3^{\text {a }}(176,7 \pm 25,3$ vs. $159,6 \pm 20,6)$ e $4^{\text {a }}$ séries $(176,1 \pm 24,7$ vs. $156,9 \pm 20,8)$; para PAD $(\mathrm{mmHg})$ na $4^{a}$ série $(99,3 \pm 11,7 v s$. $82,6 \pm 12,6)$ e para o DP (mmHg.bpm) na $4^{\text {a }}$ série $(20893,4 \pm 6215,6$ vs. 16771,3 $\pm 3981,7)$. Esses resultados indicam que a FC não seria influenciada por intervalos de recuperação de até dois minutos entre as séries. As demais variáveis, principalmente a PAS, parecem ser sensíveis ao número de séries e tempo de intervalo, o que deveria ser considerado no planejamento de programas de treinamento contra-resistência.

Palavras-chave: fisiologia cardiovascular, actividade física, saúde, treinamento contra-resistência.

\author{
${ }^{1}$ Laboratório de Atividade Física e Promoção da Saúde \\ Universidade do Estado do Rio de Janeiro \\ ${ }^{2}$ Laboratório de Ciências do Exercício \\ Universidade Federal Fluminense, Brasil
}

\begin{abstract}
Blood pressure, heart rate, and rate-pressure product in successive resistance training sets with different rest intervals

Acute cardiovascular responses during resistive exercises are an important aspect of the exercise safety. This study aimed to compare the systolic $(S B P)$ and diastolic blood pressure $(D B P)$, heart rate $(H R)$, and rate-pressure product (RPP) during four sets of eight maximal repetitions on unilateral leg extension performed with rest intervals of one (G1) and two minutes (G2). Ten healthy men with previous experience in resistance training volunteered for the study. SBP, $D B P$, and $H R$ were evaluated by continuous photoplethysmographic technique (Finapres') at rest, in the end of each set, and two minutes after the $4^{\text {th }}$ set. The two-way ANOVA showed statistical differences between G1 and G2, respectively, for SBP $(\mathrm{mmHg})$ on $2^{\text {nd }}(166.7 \pm 19.9 \mathrm{vs}$. $147.7 \pm 13.5), 3^{\text {rd }}(176.7 \pm 25.3$ vs. 159.6 \pm 20.6$)$ and $4^{\text {th }}$ set $(176.1 \pm 24.7$ vs. $156.9 \pm 20.8)$; for DBP $(\mathrm{mmHg})$ on $4^{\text {th }}$ set $(99.3 \pm 11.7$ vs. 82.6 \pm 12.6$)$ and for DP (mmHg.bpm) on $4^{\text {th }}$ set $(20893.4 \pm 6215.6$ vs. $16771.3 \pm 3981,7)$. These results suggest that $H R$ is not affected by rest intervals within two minutes. The other variables, principally SBP, seemed to be responsive to the number of sets and rest intervals period, which may be taken into account for designing resistance training programs.
\end{abstract}

Key Words: cardiovascular physiology, exercise, health, resistive training. 


\section{INTRODUÇÃO}

Um dos objectivos de um programa de actividades físicas é o aumento da força muscular, promovendo respostas que podem estar associadas à saúde e qualidade de vida, como maior eficiência em actividades quotidianas (28), declínio do risco de quedas em idosos (4), diminuição do estresse cardíaco para um determinado esforço $(7,17)$, redução dos níveis tensionais de repouso em indivíduos hipertensos (11) e diminuição dos riscos de doença cardíaca (29). O planejamento do treinamento da força envolve classicamente o controle de variáveis como tipo de contracção, número de repetições e carga mobilizada. Estudos recentes, porém, sugerem que a maximização dos seus efeitos também se relaciona ao volume do trabalho (23). Uma forma de aumentar esse volume é a condução de sessões com séries múltiplas nestes casos, os ganhos mais significativos parecem ocorrer quando são realizadas quatro séries, tanto em sujeitos treinados quanto destreinados (24). Neste contexto, é conveniente conhecer as respostas fisiológicas no decorrer das séries, destacando-se as respostas cardiovasculares devido ao impacto directo na segurança da actividade. A literatura fornece dados sobre as respostas cardiovasculares durante o exercício de força, tendo sido observadas as influências de variáveis como velocidade de movimento (12), número de séries (8) e repetições (6), percentual de carga máxima (9), tipo de contracção muscular (25), massa muscular e padrão respiratório (15), tipos de exercícios $(3,13)$ e estado de treinamento $(7,26)$.

Entretanto, são relativamente escassas as informações sobre o comportamento agudo das variáveis cardiovasculares quando se manipula o intervalo de recuperação. O Colégio Americano de Medicina do Esporte (2), por exemplo, considera suficiente um intervalo de recuperação para exercícios uniarticulares (como a extensão do joelho) entre um e dois minutos, evidenciando que o ganho de força não será comprometido. Entretanto, intervalos diferentes podem se associar a respostas cardiovasculares agudas diversas, devendo ser mais bem exploradas em relação às possibilidades de prescrição. Assim, o objectivo do presente estudo foi observar as pressões arteriais sistólica (PAS) e diastólica (PAD) e frequência cardíaca (FC), assim como estimar o duplo-produto (DP), durante quatro séries de oito repetições máximas (8 RM) no movimento de extensão unilateral do joelho, executadas com intervalos de um e dois minutos.

\section{MÉTODOS}

\section{Selecção dos sujeitos}

Participaram do estudo 10 homens (idade $22 \pm 3$ anos; massa corporal $70 \pm 11 \mathrm{~kg}$; estatura $171 \pm 9 \mathrm{~cm}$ ), experientes no treinamento de força, recrutados em instituição de ensino superior de educação física, aparentemente saudáveis. Todos foram voluntários e assinaram termo de consentimento, conforme resolução $\mathrm{n}^{\circ}$ 196/96 do Conselho Nacional de Saúde do Brasil para experimentos com humanos. Os participantes foram orientados a não ingerir cafeína ou álcool $24 \mathrm{~h}$ antes da colecta de dados e consumir alimentos até três horas antes da execução dos testes. Além disso, foram considerados como critérios de exclusão uso de substâncias ergogênicas e medicamentos que afectassem as respostas cardiovasculares, comprometimentos articulares e PAS e PAD em repouso superiores, respectivamente, a 139 e $89 \mathrm{mmHg}$.

\section{Colecta de dados}

Antes da coleta de dados propriamente dita, foi realizado o procedimento para estabelecer a carga de 8 RM na extensão unilateral do joelho do membro dominante, no equipamento cadeira extensora. A posição inicial foi caracterizada pelo joelho flectido em ângulo correspondente a $90^{\circ}$ e a posição final estabelecida com a extensão total do joelho, com as fases concêntrica e excêntrica realizadas em dois segundos cada. A determinação da carga foi realizada em dois dias. Em cada dia, estabeleceu-se até três tentativas para atingir a carga, com intervalo entre elas de pelo menos 5 minutos. Considerou-se como válida a carga do segundo dia que não fosse superior a $5 \%$ daquela obtida no dia anterior $(r=0,97)$. Além disso, no segundo dia de teste, antes da determinação da carga, as variáveis PAS, PAD e FC foram aferidas após o sujeito permanecer sentado por aproximadamente 10 minutos em posição confortável em ambiente calmo.

Após a determinação da carga, o protocolo experimental foi conduzido em mais dois dias, não consecutivos. No primeiro dia, foram aferidas novamente PAS, PAD e FC em repouso e os participantes foram aleatoriamente divididos para realizarem quatro séries de 8 RM com intervalo de um ou dois minutos entre as séries. Caso pelo menos uma das variáveis cardiovasculares apresentasse discrepâncias em relação ao valor observado no primeiro dia, o exercício não era realizado e solicitava-se uma nova visita. 
Finalmente, no último dia, o mesmo procedimento foi conduzido, sendo que os sujeitos que realizaram o exercício com um minuto de descanso entre as séries, o fizeram com dois minutos de intervalo e vice-versa. Todos os dados foram coletados entre 14:00 e 16:00 h.

\section{Medida da pressão arterial}

A pressão arterial e a $\mathrm{FC}$ em repouso, durante o exercício de força e na fase de recuperação foram aferidas por fotopletismografia (Finapres ${ }^{\mathrm{TM}} 2350$, Ohmeda, WI, USA). Embora não seja o padrão-ouro para medidas da pressão arterial, fornece leitura contínua de PAS, PAD e FC, sendo, no caso do exercício de força, o método não-invasivo mais indicado para medir tais variáveis (21). A medida é obtida pela regulação pneumática de um cuff acoplado no dedo médio da mão esquerda sobre a artéria digital. $\mathrm{O}$ registro expresso em mmHg é a captação da distensão da parede vascular pelo cuff. Foi solicitado aos participantes que flexionassem o cotovelo, de modo a aproximar a mão do tórax, a fim de evitar que os valores registrados fossem contaminados pela acção gravitacional, caso o braço permanecesse estendido. Os sujeitos também foram instruídos a não contrair ou movimentar o braço, a mão e os dedos onde o cuff estava acoplado, assim como não realizar a manobra de Valsalva. Para melhor familiarização com a técnica de medida da PA durante o exercício, os sujeitos realizaram o teste de $8 \mathrm{RM}$ do primeiro dia com a mão na posição estipulada, ou seja, próxima ao tórax e sem qualquer tipo de contracção. A medida em repouso foi realizada com o sujeito sentado na cadeira extensora, para que não houvesse qual- quer movimentação corporal entre os registros feitos antes e durante o exercício. Durante as séries, os valores registrados foram obtidos imediatamente no término da execução das repetições previstas. Após o término da última série, o sujeito permaneceu na mesma posição durante dois minutos, para que se acompanhasse o processo de recuperação.

\section{Tratamento dos dados}

Os dados foram analisados por meio de uma ANOVA factorial de duas entradas (intervalo de recuperação e número de séries). As variáveis que se mostraram significativas foram tratadas por contraste, a fim de verificar diferenças entre os valores observados nas condições pré e pós-exercício, e entre esses valores e os obtidos em cada uma das séries. Adoptou-se como significância estatística $\mathrm{p}<0,05$. O tratamento dos dados foi realizado no software Statistica $^{\circledR}$ (versão 5.5, Statsoft, OK, USA).

\section{RESULTADOS}

As Tabelas 1 e 2 mostram os valores observados no repouso, em cada série e no período pós-exercício da extensão do joelho realizada com um (G1) e dois minutos (G2) de intervalo de recuperação entre as séries, respectivamente. Não se verificaram diferenças entre os valores obtidos em repouso para todas as variáveis em ambos os grupos, assim como entre o repouso e cada minuto pós-exercício. Em todas as séries, as variáveis mostraram-se significativamente mais elevadas que os valores observados no repouso e no pós-exercício, independentemente do intervalo de recuperação. Apenas as variáveis PAS e PAD em G1 exibiram diferenças significativas intra-séries.

Tabela 1. Valores absolutos das variáveis cardiovasculares em cada série da extensão do joelho realizada com intervalos de recuperação de um minuto (G1) (média \pm desvio padrão).

\begin{tabular}{|c|c|c|c|c|}
\hline & $\begin{array}{l}\text { Pressão arterial } \\
\text { sistólica (mmHg) }\end{array}$ & $\begin{array}{c}\text { Pressão arterial } \\
\text { diastólica (mmHg) }\end{array}$ & $\begin{array}{l}\text { Frequência cardiaca } \\
\text { (bpm] }\end{array}$ & $\begin{array}{l}\text { Duplo-produto } \\
\text { (mmHg.bpm] }\end{array}$ \\
\hline Repouso & $123,1 \pm 7,3$ & $59,0 \pm 6,4$ & $80,2 \pm 17,3$ & $9870,3 \pm 2250,3$ \\
\hline 1asérie & $152,9 \pm 21,5^{*}$ & $76,7 \pm 13,3^{*}$ & $113,9 \pm 24,6^{*}$ & $17544,4 \pm 4953,3^{*}$ \\
\hline Zasérie & $166,7 \pm 19,9^{*}$ & $85,6 \pm 10,6^{*}$ & $113,1 \pm 18,4^{*}$ & $19044,3 \pm 4868,6^{*}$ \\
\hline 3asérie & $176,7 \pm 25,3^{*}, 1$ & $95,7 \pm 12,9^{*}, 1,2$ & $109,2 \pm 19,8^{*}$ & $19697,0 \pm 5969,5^{*}$ \\
\hline 4asérie & $176,1 \pm 24,7^{*}, 1$ & $99,3 \pm 11,7^{*}, 1,2$ & $116,7 \pm 21,5^{*}$ & $20893,4 \pm 6215,6^{*}$ \\
\hline 1 min pós-exercício & $131, ? \pm 18,0$ & $64,1 \pm 9,4$ & $89,9 \pm 21,6$ & $11897,6 \pm 3545,6$ \\
\hline 2 min pós-exercício & $120,3 \pm 11,8$ & $62,0 \pm 9,6$ & $86,5 \pm 19,1$ & $10472,3 \pm 2781,9$ \\
\hline
\end{tabular}

* diferença significativa em relação ao repouso e às medidas pós-exercício;

1-diferença significativa em relação à primeira série; 2-diferença significativa em relação à segunda série 
Tabela 2. Valores absolutos das variáveis cardiovasculares em cada série da extensão do joelho realizada com intervalos de recuperação de dois minutos (G2) (média \pm desvio padrão).

\begin{tabular}{l|c|c|c|c} 
& $\begin{array}{c}\text { Pressão arterial } \\
\text { sistólica }(\mathrm{mmHg})\end{array}$ & $\begin{array}{c}\text { Pressão arterial } \\
\text { diastólica }(\mathrm{mmHg})\end{array}$ & $\begin{array}{c}\text { Freqüência } \\
\text { cardíaca } \text { (bpm) }\end{array}$ & $\begin{array}{c}\text { Duplo-produto } \\
\text { (mmHg.bpm] }\end{array}$ \\
\hline Repouso & $118,5 \pm 8,1$ & $59,9 \pm 8,6$ & $70,7 \pm 10,2$ & $8384,7 \pm 1444,8$ \\
1 asérie & $147,4 \pm 14,3^{*}$ & $77,5 \pm 11,9^{*}$ & $106,8 \pm 19,1^{*}$ & $15893,6 \pm 4181,8^{*}$ \\
2asérie & $147,7 \pm 13,5^{*}$ & $86,6 \pm 17,0^{*}$ & $107,4 \pm 17,8^{*}$ & $15914,4 \pm 3450,0^{*}$ \\
3asérie & $159,6 \pm 20,6^{*}$ & $86,4 \pm 13,8^{*}$ & $107,8 \pm 17,7^{*}$ & $17408,2 \pm 4707,6^{*}$ \\
4asérie & $156,4 \pm 20,8^{*}$ & $82,6 \pm 12,6^{*}$ & $106,2 \pm 14,4^{*}$ & $16771,3 \pm 3981,7^{*}$ \\
1 min pós-exercício & $127,4 \pm 8,8$ & $66,3 \pm 7,9$ & $77,6 \pm 11,9$ & $9842,3 \pm 1440,7$ \\
2 min pós-exercício & $117,6 \pm 9,5$ & $63,1 \pm 8,4$ & $76,0 \pm 13,3$ & $8937,7 \pm 1769,7$
\end{tabular}

* diferença significativa em relação ao repouso e às medidas pós-exercício.

O tratamento estatístico mostrou que as variáveis PAS $(p=0,0001)$, PAD $(p=0,02)$ e DP $(p=0,002)$ sofreram influência significativa do número de séries e intervalo de recuperação. As diferenças quanto ao intervalo de recuperação são exibidas nas Figuras 1, 2 e 3 . As discrepâncias mais importantes foram observadas para a PAS, uma vez que apenas na primeira série os valores foram semelhantes (Figura 1). Já em relação à PAD (Figura 2) e ao DP (Figura 3), a única diferença significativa ocorreu na quarta série. Não foram observadas diferenças para a FC.

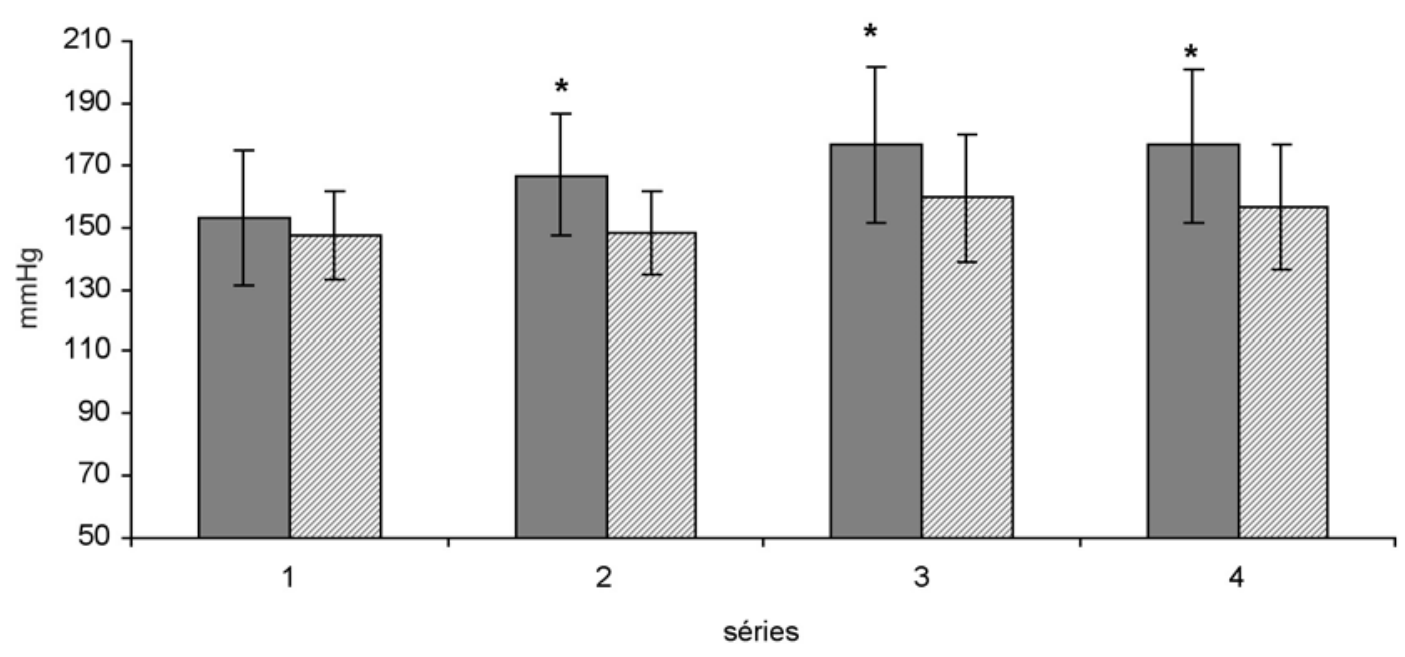

Figura 1. Comportamento da pressão arterial sistólica em cada série da extensão do joelho realizada com intervalos de recuperação de um [G1] e dois minutos (G2). 


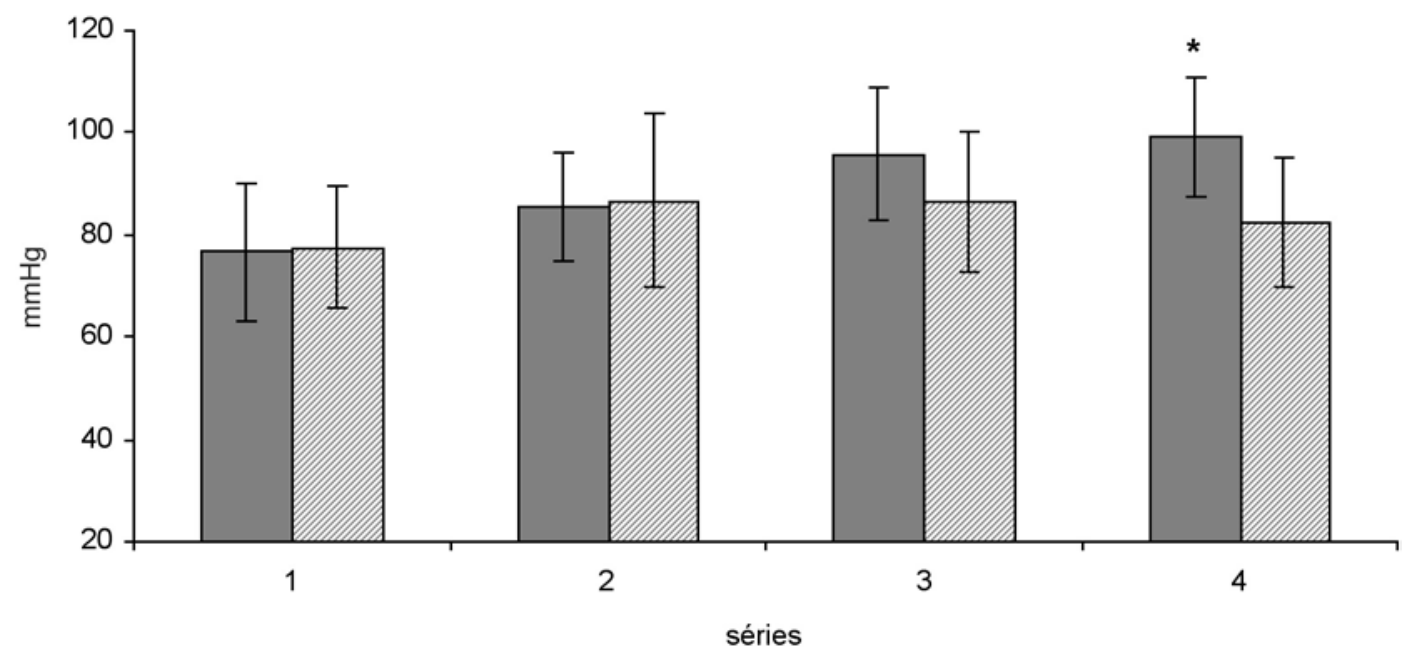

Figura 2. Comportamento da pressão arterial diastólica em cada série da extensão do joelho realizada com intervalos de recuperação de um (G1) e dois minutos (G2).

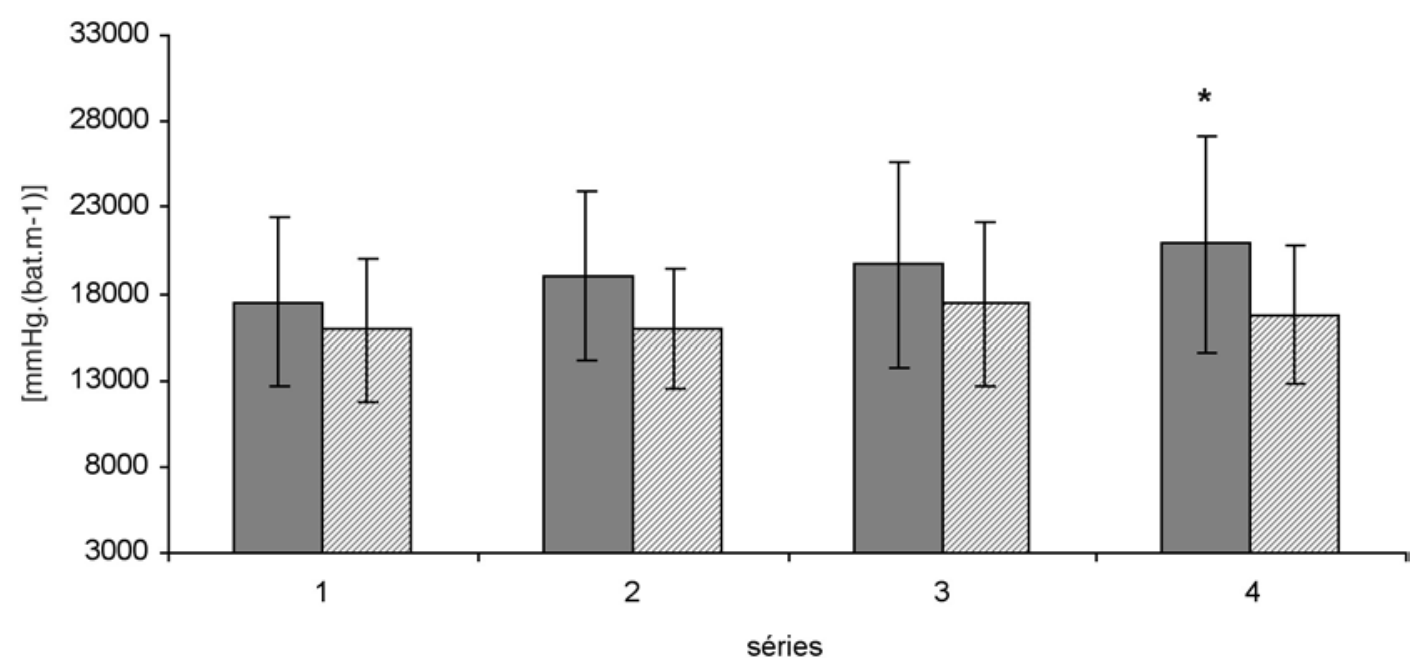

Figura 3. Comportamento do duplo-produto em cada série da extensão do joelho realizada com intervalos de recuperação de um (G1) e dois minutos (G2). 


\section{DISCUSSÃO}

O estudo visou comparar as respostas cardiovasculares durante o exercício de força, realizado com diferentes intervalos de recuperação em quatro séries consecutivas de 8 RM. Embora os resultados mostrem que todas as variáveis tenderam a aumentar significativamente durante o esforço, é interessante notar que, em pelo menos uma das séries, os valores de PAS, PAD e DP na extensão unilateral do joelho mostraram-se significativamente mais elevados quando $o$ exercício foi realizado com intervalo de um minuto. Provavelmente, a fadiga periférica pode estar na origem desse comportamento. Embora todos os sujeitos conseguissem realizar o número de repetições estipulado em cada série, o tempo de recuperação mais curto entre elas pode ter contribuído para elevar o estresse fisiológico associado àquela intensidade de trabalho. Isso poderia ser atribuído a dois principais mecanismos: um de origem central e outro periférico. O mecanismo central envolve a irradiação de impulsos do córtex motor para o centro de controle cardiovascular. Este mecanismo está diretamente relacionado com a sensação subjetiva de esforço, o que pode consistir em estímulo considerável para elevar as respostas cardiovasculares quando o exercício é intenso (20). Já o mecanismo periférico consiste em uma via de reflexo com bases ainda pouco esclarecidas (5). Esse mecanismo é originado na liberação de metabolitos dos músculos que estão ativos (como potássio e ácido lático, por exemplo), aumentando a osmolaridade do líquido intersticial (19). A liberação dessas substâncias podem ativar terminações nervosas sensíveis a alterações químicas (quimiorreceptores), as quais fazem feedback com o centro de controle cardiovascular, aumentando a pressão arterial $(19,20)$. Além disso, o aumento da pressão arterial pode ser influenciado pelo número de unidades motoras solicitadas durante o exercício. Nesse caso, o mecanismo de ajuste cardiovascular seria percebido por estruturas sensíveis ao aumento da força e da velocidade de movimento (mecanorreceptores) que, assim como os quimiorreceptores, informam o centro de controle cardiovascular sobre a necessidade de modificar as respostas cardiovasculares (22). O aumento das unidades motoras e, conseqüentemente, da massa muscular também pode aumentar a pressão arterial, devido à compressão muscular no leito vascular que bloqueia parcialmente a circulação (15).

Outra possibilidade que se deve considerar, ainda que marginal, poderia ser uma maior propensão para a manobra de Valsalva (15) que, mesmo sendo desencorajada, não pôde ser totalmente controlada.

Embora a realização da manobra de Valsalva durante o exercício de força possa contribuir para elevar a pressão arterial (14), durante solicitações muito intensas a manobra de Valsava é quase inevitável, pois estabiliza o tronco e favorece a execução (16). Assim, no presente estudo, poder-se-ia especular que o exercício realizado com intervalo mais prolongado entre as séries pareceu permitir que um novo estímulo fosse realizado sem interferir demasiadamente nas respostas pressóricas, provavelmente devido a uma menor ação dos quimiorreceptores e mecanorreceptores e menor propensão à manobra de Valsalva. Os estudos que se aprofundaram na observação do comportamento da pressão arterial e FC associadas ao intervalo de recuperação entre as séries do exercício de força, controlando todas as variáveis envolvidas, são relativamente poucos. No experimento de Meyer et al. (18), por exemplo, os autores compararam a PAS, PAD e FC em situações distintas de intensidade e intervalo de recuperação em exercícios para membros inferiores. Os autores não identificaram diferenças significativas para as variáveis cardiovasculares, ao compararem a execução de uma série com 60 segundos de duração, carga de $65 \%$ de 1 RM e intervalo entre os exercícios de 60 segundos, e outra série com duração de 30 segundos, carga de $85 \%$ de 1 RM e descanso de 45 segundos. Isso poderia estar relacionado à manipulação simultânea da carga, tempo de estímulo e período de recuperação. Do primeiro para o segundo momento, a carga e o tempo de recuperação aumentaram, respectivamente, cerca de $24 \%$ e $50 \%$, enquanto que o tempo de estímulo reduziu-se em 50\%. Desse modo, diferenças não foram observadas mas, provavelmente, as respostas cardiovasculares comportar-se-iam de outra maneira, caso uma das três variáveis descritas não fosse manipulada. Como no presente estudo a única variável manipulada foi o tempo de recuperação, já que séries, repetições e velocidade de movimento mantiveram-se constantes em ambos os grupos, pôde-se compreender melhor a influência isola- 
da do intervalo de descanso sobre as variáveis cardiovasculares.

Independentemente do intervalo de recuperação, parece existir um efeito cumulativo quanto à elevação das respostas cardiovasculares em relação ao número de séries consecutivas realizadas. Gotshall et al. (8) observaram o comportamento da pressão arterial em séries, repetições e intervalos constantes. Identificou-se que, ao término de cada uma das três séries de 10 RM no leg-press bilateral, a PAS estava significativamente mais elevada em relação à série anterior. Entretanto, os autores utilizaram intervalo de recuperação fixo em três minutos e o movimento foi realizado em três segundos para cada uma das fases concêntrica e excêntrica. Desse modo, o tempo de tensão para cada série situou-se em torno de um minuto. Isso, somado à maior massa muscular envolvida no exercício leg-press, poderia explicar a elevação identificada para a PAS. No presente estudo, utilizou-se um tempo de contracção de dois segundos em cada fase do movimento, com 32 segundos de tensão total por série. Além disso, a extensão unilateral do joelho solicita menos músculos que o leg-press. Desse modo, mesmo no grupo que descansou menos (G1), o aumento da pressão arterial não se revelou progressivo à medida que se realizavam as séries.

No presente experimento, não se observaram diferenças na FC associadas ao intervalo de recuperação. A elevação da FC durante o exercício de força pode ser importante quando o número de repetições é máximo e a carga submáxima $(6,15)$. Isso foi relatado por Fleck e Dean (7), em estudo no qual a FC não apresentou modificações significativas em 50 , 60 , 70 e $90 \%$ da carga máxima na extensão unilateral do joelho e no desenvolvimento unilateral em fisiculturistas. Provavelmente, a velocidade de movimento foi maior com cargas mais leves, não gerando diferenças entre o tempo total de tensão, já que se verificou que a FC eleva-se em relação ao tempo de exposição ao estímulo (27).

Desse modo, o tempo de tensão parece ser um dos factores mais importantes para o incremento dessa variável e está relacionado à velocidade de movimento. Isso foi observado no experimento de Kleiner et al. (12), no qual se verificou que a velocidade mais rápida $\left(200^{\circ} . \mathrm{seg}^{-1}\right)$ da extensão do joelho até à fadiga proporcionou maior incremento da FC e maior número de repetições (cerca de quatro vezes) que $50^{\circ}$. $\mathrm{seg}^{-1}$. Ou seja, a velocidade quadruplicada ocasionou repetições aumentadas na mesma proporção, porém com valores mais elevados da FC. A princípio, velocidades mais rápidas estão associadas a cargas mais leves, o que favorece um maior período de exposição à contracção muscular. Do mesmo modo, Hunter et al. (10) verificaram que o exercício de força realizado na velocidade denominada 'tradicional' induziria FC médias significativamente maiores que a técnica denominada 'super lenta', com velocidade de movimento extremamente baixa.

No presente estudo, não foram identificadas diferenças para a FC em relação aos intervalos de recuperação para G1 e G2 (Tabelas 1 e 2, respectivamente), sugerindo que intervalos de um ou dois minutos não repercutiriam sobre a elevação da FC ao término de cada série. Isso pode ser, em parte, explicado pelo facto de a tendência de elevação da FC ser 'freada' por um tempo relativamente curto de contracção (3). Ilustrando esse facto, no presente experimento, a FC apresentou elevação na primeira série de aproximadamente $44 \%$ para G1 e $53 \%$ para G2, após 32 segundos de contracção muscular, sem variar significativamente ao longo do trabalho.

$\mathrm{O}$ facto de a FC não variar pode ter influenciado o comportamento do DP. Embora essa variável não seja válida para estimar o consumo de oxigénio pelo miocárdio em actividades de alta intensidade e curta duração, pode ser considerada como o melhor indicador de solicitação cardíaca durante o exercício de força (1). No presente estudo, o DP somente apresentou diferença significativa entre G1 e G2 na última série. Entretanto, como o DP é estimado pela multiplicação da PAS pela FC, uma possibilidade pode ser apontada: o tratamento estatístico de cada variável dependente mostrou que a PAS associava-se significativamente $(\mathrm{p}<0,05)$ ao número de séries e intervalo de recuperação, enquanto a $\mathrm{FC}$ não estava associada a nenhum deles. Nesse sentido, é possível que a quantificação isolada da FC, sem mensuração concomitante dos valores de PA, não constitua uma forma segura de apreciar o estresse cardiovascular em exercícios de força.

Finalmente, ao término do exercício, todas as variáveis cardiovasculares exibiram valores próximos aos 
de repouso, independentemente do intervalo de recuperação sugerido. Uma das explicações para esses resultados pode estar relacionada ao rápido declínio dos valores das variáveis cardiovasculares ao fim do exercício, chegando-se em poucos segundos a patamares próximos aos valores de repouso. De facto, a recuperação total da pressão arterial pode ocorrer em aproximadamente 10 segundos, mesmo em face de um aumento importante durante a actividade (15). Essa redução pode estar relacionada à abrupta perfusão sanguínea pela vasodilatação da musculatura que estava ocluída e pelos mecanismos reflexos barorreceptor e cardiopulmonar, em resposta à elevação demasiada da pressão arterial ao final da última repetição (15). Desse modo, independentemente do intervalo de recuperação entre as séries, a redução da PAS, PAD e FC ao final do exercício de força parece não ser influenciada pelo seu valor final.

\section{CONCLUSÃO}

O presente estudo teve como objectivo verificar as respostas cardiovasculares durante um exercício de força realizado segundo recomendações atuais de prescrição, tanto para séries $(23,24)$ quanto para o intervalo de recuperação (2). Os resultados indicaram que o mesmo exercício, no caso a extensão unilateral do joelho, poderia apresentar respostas cardiovasculares agudas diferentes quando se manipulou o intervalo de recuperação entre as séries. Nesse sentido, partindo das informações bibliográficas de que intervalos diferentes podem representar ganhos de força semelhantes (2), seria interessante, em caso de necessidade de controle cardiovascular durante o exercício, optar pelo intervalo mais prolongado. Contudo, é importante comentar que o período de descanso de dois minutos como provável segurança cardiovascular não deve ser generalizado para todas as situações de treinamento de força. Em outros tipos de exercícios, como os que envolvem várias articulações (e conseqüentemente mais grupos musculares), o intervalo considerado como satisfatório no presente estudo poderia não ser o mesmo. Para além disso, não identificamos na literatura um limite a partir do qual as respostas cardiovasculares representariam riscos no exercício de força. Esse facto nos impossibilita de discutir se os valores das respostas cardiovasculares alcançados no presente estudo, inde- pendentemente do intervalo de recuperação entre as séries, estariam de acordo com a segurança do indivíduo. Aparentemente, a questão dos limites de segurança é individual e maiores especulações nesse sentido fugiriam do propósito da nossa investigação. Entretanto, apesar das evidências apontadas por este estudo, a influência do intervalo de recuperação entre séries de exercícios de força sobre as respostas cardiovasculares agudas, precisaria de estudos adicionais para ser mais bem definida. Ainda são insuficientes as informações sobre tal comportamento em sujeitos cardiopatas ou hipertensos, o que abre caminhos de investigação. O mesmo se aplica a outros tipos de exercício, como os que envolvem várias articulações, nos quais o intervalo de recuperação poderia ser maior que dois minutos. Dessa forma, a análise de outros exercícios, modos de execução, intervalos de recuperação e estado de treinamento, por exemplo, contribuiria para o melhor entendimento do comportamento cardiovascular durante o processo de treinamento de força.

\author{
Agradecimento \\ Estudo parcialmente financiado pelo Conselho \\ Nacional de Desenvolvimento Científico e \\ Tecnológico (CNPq), sob forma de bolsa de \\ Mestrado (Marcos Polito) e Produtividade em \\ Pesquisa (Paulo Farinatti).
}

\section{CORRESPONDÊNCIA}

\section{Marcos D. Polito}

Laboratório de Atividade Física e Promoção da Saúde Rua São Francisco Xavier, 524, sala 8133, bloco F 20550-013 - Rio de Janeiro - RJ

BRASIL

mdpolito@uol.com.br 


\section{REFERÊNCIAS BIBLIOGRÁFICAS}

1. American College of Sports Medicine (2000). ACSM's Guidelines for Exercise Testing and Prescription. 6 ed. Baltimore: Williams \& Wilkins.

2. American College of Sports Medicine (2002). Position stand on progression models in resistance training for healthy adults. Med Sci Sports Exerc 34: 364-380.

3. Benn SJ, McCartney N, McKelvie RS (1996). Circulatory responses to weight lifting, walking, and stair climbing in older males. J Am Geriatr Soc 44: 121-125.

4. Buchner DM, Cress ME, de Lateur BJ, Esselman PC, Margherita AJ, Price R, Wagner EH (1997). The effect of strength and endurance training on gait, balance, fall risk, and health services use in community-living older adults. $J$ Gerontol A Biol Sci Med Sci 52A: M218-224.

5. Carrington CA, Ubolsakka C, White MJ (2003). Interaction between muscle metaboreflex and mechanoreflex modulation of arterial baroreflex sensitivity in exercise. J Appl Physiol 95: 43-8.

6. Farinatti PTV, Assis BFC (2000). Estudo de freqüência cardíaca, pressão arterial e duplo-produto em exercícios contraresistência e aeróbio contínuo. Rev Bras Ativ Fis Saúde 5: 5-16.

7. Fleck SJ, Dean LS (1987). Resistance-training experience and the pressor response during resistance exercise. J Appl Physiol 63: 116-120.

8. Gotshall R, Gootman J, Byrnes W, Fleck S, Valovich T (1999). Noninvasive characterization of the blood pressure response to the double-leg press exercise. JEPonline 2: 1-6.

9. Haslam DRS, McCartney N, McKelvie RS, MacDougall JD (1988). Direct measurements of arterial blood pressure during formal weightlifting in cardiac patients. $J$ Cardiopulm Rehabil 8: 213-225.

10. Hunter GR, Seelhorst D, Snyder S (2003). Comparison of metabolic and heart rate responses to super slow vs traditional resistance training. J Strength Cond Res 17: 76-81.

11. Kelley GA, Kelley KS (2000). Progressive resistance exercise and resting blood pressure: a meta-analysis of randomized controlled trials. Hypertension 35: 838-843.

12. Kleiner DM, Blessing DL, Mitchell JW, Davis WR (1999). A description of the acute cardiovascular responses to isokinetic resistance at three different speeds. J Strength Cond Res 13: 360-366.

13. Leite TC, Farinatti PTV (2003). Heart rate, systolic blood pressure, and rate pressure product in different resistive exercises for similar muscle groups. Rev Bras Fisiol Exerc 2: 68-88.

14. Linsenbardt ST, Thomas TR, Madsen RW (1992). Effect of breathing techniques on blood pressure response to resistance exercise. Br J Sports Med 26: 97-100.

15. MacDougall JD, Tuxen D, Sale DG, Moroz JR, Sutton JR (1985). Arterial blood pressure response to heavy resistance exercise. J Appl Physiol 58: 785-790.

16. MacDougall JD, McKelvie RS, Moroz DE, Sale DG, McCartney N, Buick F (1992). Factors affecting blood pressure during heavy weight lifting and static contractions. J Appl Physiol 73: 1590-7.

17. McCartney N, McKelvie RS, Martin J, Sale DG, MacDougall JD (1993). Weight-training-induced attenuation of the circulatory response of older males to weight lifting. J Appl Physiol 74: 1056-1060.
18. Meyer K, Kardos A, Samek L, Lehmann M, Kurz K, Caspar U, Droste C, Betz P, Weidemann H, Roskamm H (1992). Interval resistance exercise in comparison with bicycle ergometry stress. Studies with resistance endurance training in coronary patients. $Z$ Kardiol 81: 531-537.

19. Mitchell JH, Schibye B, Payne FC, Saltin B (1981). Response of arterial blood pressure to static exercise in relation to muscle mass, force development, and electromyographic activity. Circ Res 48: 170-175.

20. Mitchell JH, Payne FC, Saltin B, Schibye B (1980). The role of muscle mass in the cardiovascular response to static contractions. J Physiol London 309: 45-54.

21. Polito MD, Farinatti PTV (2003). Considerações sobre a medida da pressão arterial em exercícios contra-resistência. Rev Bras Med Esporte 9: 25-33.

22. Prabhakar NR, Peng YJ (2004). Peripheral chemoreceptors in health and disease. J Appl Physiol 96: 359-366.

23. Rhea MR, Alvar BA, Burkett LN (2002). Single versus multiple sets for strength: a meta-analysis to address the controversy. Res Q Exerc Sport 73: 485-488.

24. Rhea MR, Alvar BA, Burkett LN, Ball SD (2003). A metaanalysis to determine the dose response for strength development. Med Sci Sports Exerc 35: 456-464.

25. Sale DG, Moroz DE, McKelvie RS, MacDougall JD, McCartney N (1993). Comparison of blood pressure response to isokinetic and weight-lifting exercise. Eur J Appl Physiol Occup Physiol 67: 115-120.

26. Sale DG, Moroz DE, McKelvie RS, MacDougall JD, McCartney N (1994). Effect of training on the blood pressure response to weight lifting. Can J Appl Physiol 19: 60-74.

27. Smolander J, Aminoff T, Korhonen I, Tervo M, Shen N, Korhonen O, Louhevaara V (1998). Heart rate and blood pressure responses to isometric exercise in young and older men. Eur J Appl Physiol 77: 439-444.

28. Suzuki T, Bean JF, Fielding RA (2001). Muscle power of the ankle flexors predicts functional performance in community dwelling older women. J Am Geriatr Soc 49: 1161-1167.

29. Tanasescu M, Leitzmann MF, Rimm EB, Willett WC, Stampfer MJ, Hu FB (2002). Exercise type and intensity in relation to coronary heart disease in men. J Am Med Assoc 288: 1994-2000. 